\title{
Table S1. Oligonucleotides used in this study.
}

For mutagenesis primers, the mutated nucleotide is in bold. The 3' ends of the primers

(underlined) were designed to anneal to plasmid pEPkan-S, a plasmid used as a template for a

PCR reaction that yields an amplification product containing an I-SceI site located upstream of the aphAI gene conferring kanamycin resistance (Tischer BK, von Einem J, Kaufer B,

Osterrieder N (2006) Two-step red-mediated recombination for versatile high-efficiency

markerless DNA manipulation in Escherichia coli. BioTechniques 40: 191-197). Sequencing

primers for pol (ORF30) were first used to amplify a $466 \mathrm{bp}$ fragment of pol from BAC DNA

and also from virus co-cultured from equine PBMC and RK13 cells in the infection experiments.

PCR products were purified using the Qiagen PCR cleanup kit prior to sequencing using the

same primers. Oligonucleotides for measurement of murine $i N O S$ and equine $\beta 2-M$ gene copies

by qPCR were designed using Primer Express 2.0. The probes are fluorescently labeled on the

5' end with VIC, and have a non-fluorescent minor groove-binding (MGB) quencher.

\begin{tabular}{|c|c|l|}
\hline Purpose & Description & Sequence (5'-3') \\
\hline mutagenesis & $\begin{array}{c}\text { Ab4-ORF30_ } \\
\text { D752N_F }\end{array}$ & $\begin{array}{l}\text { CGATGAAGTGGATCTGGCCGGGCTTCAACCATCCGTCAACT } \\
\text { ACTCGACGTTCGAGTAGGGATAACAGGGTAATCGATTT }\end{array}$ \\
\hline mutagenesis & $\begin{array}{c}\text { Ab4-ORF30_ } \\
\text { D752N_R }\end{array}$ & $\begin{array}{l}\text { AAATAACTTTTGGTCACCACCTCGAACGTCGAGTAGTTGA } \\
\text { CGGATGGTTGAAGCCGCCAGTGTTACAACCAATTAACC }\end{array}$ \\
\hline mutagenesis & $\begin{array}{c}\text { Ab4-ORF30_ } \\
\text { N752D_F }\end{array}$ & $\begin{array}{l}\text { CGATGAAGTGGATCTGGCCGGGCTTCAACCATCCGTCGACT } \\
\text { ACTCGACGTTCGAGTAGGGATAACAGGGTAATCGATTT }\end{array}$ \\
\hline mutagenesis & $\begin{array}{c}\text { Ab4-ORF30_ } \\
\text { N752D_R }\end{array}$ & $\begin{array}{l}\text { AAATAACTTTTGGTCACCCACCTCGAACGTCGAGTAGTCGA } \\
\text { CGGATGGTTGAAGCCGCCAGTGTTACAACCAATTAACC }\end{array}$ \\
\hline mutagenesis & $\begin{array}{c}\text { pTM1-ORF30_ } \\
\text { D752N_F }\end{array}$ & CAACCATCCGTCAACTACTCGACGTTCGAGG \\
\hline mutagenesis & $\begin{array}{c}\text { pTM1-ORF30_ } \\
\text { D752N_R }\end{array}$ & CCTCGAACGTCGAGTAGTTGACGGATGGTTG \\
\hline sequencing & ORF30_F & GCTACTTCTGAAAACGGAGGC \\
\hline sequencing & ORF30_R & CTATCCTCAGACACGGCAACA \\
\hline qPCR & murine $i N O S \_F$ & CACTGTGTGCCAAGCCTAGGA \\
\hline qPCR & murine $i N O S$ probe & VIC-TCTGAGCGCCTCCAG-MGBNFQ \\
\hline qPCR & murine $i N O S_{-}$R & TCACAACGTGCCATCTGAAAG \\
\hline qPCR & equine $\beta 2-M \_F$ & TCTTTCAGCAAGGACTGGTCTTT \\
\hline qPCR & equine $\beta 2-M$ probe & VIC-ATCTTCTGGTCCATACTGA-MGBNFQ \\
\hline qPCR & equine $\beta 2-M \_R$ & CATCCACACCATTGGGAG TAA A \\
\hline
\end{tabular}

